\title{
Molecular dynamics simulations of free energy and conformational transition rates of calix[4]arene in chloroform
}

\author{
W. K. den Otter and W. J. Briels \\ Chemical Physics Laboratory, University of Twente, P.O. Box 217, 7500 AE Enschede, The Netherlands
}

(Received 21 May 1997; accepted 25 June 1997)

\begin{abstract}
In a previous article we introduced a reaction coordinate based on the unstable normal mode at the saddle point of the potential energy surface. We here calculate the free-energy distribution along this coordinate for the isomerization of calix[4]arene in vacuo and in chloroform using umbrella sampling, with one umbrella covering the entire range of the reaction coordinate. An excellent first guess at this umbrella is obtained by performing a normal-mode analysis at various points along the reaction path. The isomerization rate constant of this reaction is determined using the reactive flux method and is found to be in good agreement with experimental data. The rate was found to be independent of the location of the transition state, as it should be. (C) 1997 American Institute of Physics. [S0021-9606(97)50437-5]
\end{abstract}

\section{INTRODUCTION}

The forward rate constant $k_{f}$ of a reaction, i.e., the fraction of reactants turned into products per unit of time, in a solvent is a complicated function of the internal potential of the reacting solute molecule(s), the solute-solvent interactions, and the solvent-solvent interactions. Molecular dynamics (MD) simulations are perfectly well suited for the numerical analysis of such involved situations. Based on the statistical mechanical ideas discussed below, it is possible to deduce from a total of about $10 \mathrm{~ns}$ simulation time a reaction rate that is slower by many orders of magnitude. The particular reaction studied here is the isomerization of a calix[4]arene in vacuo and in chloroform.

In Eyrings transition state theory ${ }^{1}$ (TST) a hyperplane, the transition state, is introduced to split configuration space into a reactant space and a product space. This plane is characterized by $\xi\left(\left\{\mathbf{x}_{i}\right\}\right)=\xi^{\neq}$, where the reaction coordinate $\xi\left(\left\{\mathbf{x}_{i}\right\}\right)$ is a function of the atomic coordinates. Product space and reactant space are defined by $\xi>\xi^{\neq}$and $\xi<\xi^{\neq}$, respectively. The forward rate constant is calculated as the instantaneous product-bound flux through the transition state, normalized by the population of the reactant space: ${ }^{2}$

$$
k_{f}^{\mathrm{TST}}=\frac{\left\langle\delta\left[\xi(0)-\xi^{\ddagger}\right] \dot{\xi}(0) \theta[\dot{\xi}(0)]\right\rangle}{\left\langle\theta\left[\xi^{\neq}-\xi(0)\right]\right\rangle} .
$$

Here the angular brackets denote a canonical average and $\theta$ is the Heaviside function.

The TST expression is conveniently rewritten as

$$
k_{f}^{\mathrm{TST}}=\frac{\left\langle\delta\left[\xi(0)-\xi^{\ddagger}\right] \dot{\xi}(0) \theta[\dot{\xi}(0)]\right\rangle}{\left\langle\delta\left[\xi(0)-\xi^{\ddagger}\right]\right\rangle} \frac{\left\langle\delta\left[\xi(0)-\xi^{\ddagger}\right]\right\rangle}{\left\langle\theta\left[\xi^{\neq}-\xi(0)\right]\right\rangle} .
$$

The second term on the right-hand side is the probability of finding the molecule at the transition state, $P\left(\xi^{\neq}\right)$, divided by the probability for the molecule to be in reactant space, $P_{R}=\int_{-\infty}^{\xi^{\ddagger}} P(\xi) d \xi$. We will discuss two techniques for the calculation of the probability distribution $P(\xi)$ : normalmode analysis in Sec. II B, and umbrella sampling in Sec.
II C. The first term on the right-hand side of Eq. (1.2), the average positive velocity of the reaction coordinate at the transition state, will be addressed in Sec. II D. The particular reaction coordinate that we will use is discussed in Sec. II A. Numerical results for the probability function and the TST isomerization rate of a calix[4]arene in vacuo and in chloroform are presented in Sec. III.

Transition state theory, by focusing on the instantaneous forward flux, neglects the fact that some fraction of this flux will recross the transition state shortly (order of a picosecond) after having crossed it. Likewise, the forward flux also contains a contribution of "product molecules" that have crossed the transition state with a negative velocity shortly before they recross with a positive velocity. These recrossings are the result of the normal dynamics of a reacting molecule, induced by both the internal interactions of the molecule and its interactions with the solvent. To a macroscopic observer, though, these rapid recrossings are invisible and irrelevant. He defines the rate by the number of molecules that are in the reactant well at time 0 and in the product well at time $t$, normalized by the overall number of molecules in the reactant well at time 0 . Only if there are no recrossings will his rate equal the TST rate, otherwise TST overestimates the true rate.

In the reactive flux method $(\mathrm{RF})$ the aforementioned macroscopic definition of the rate is related to the microscopic behavior of a single molecule in a solvent by means of Onsager's regression hypothesis. ${ }^{3,4}$ The resulting rate expression is

$$
k_{f}^{\mathrm{RF}}(t)=\frac{\left\langle\delta\left[\xi(0)-\xi^{\ddagger}\right] \dot{\xi}(0) \theta\left[\xi(t)-\xi^{\ddagger}\right]\right\rangle}{\left\langle\theta\left[\xi^{\ddagger}-\xi(0)\right]\right\rangle} .
$$

In comparison with TST, attention has shifted from those molecules that cross the transition state with a positive velocity to those molecules that actually end up in the product space at some time $t$ after crossing the transition state in whatever direction. The rate is often expressed as

$$
k_{f}^{\mathrm{RF}}(t)=\kappa(t) k_{f}^{\mathrm{TST}},
$$




$$
\kappa(t)=\frac{\left\langle\delta\left[\xi(0)-\xi^{\ddagger}\right] \dot{\xi}(0) \theta\left[\xi(t)-\xi^{\ddagger}\right]\right\rangle}{\left\langle\delta\left[\xi(0)-\xi^{\ddagger}\right] \dot{\xi}(0) \theta[\dot{\xi}(0)]\right\rangle},
$$

where $\kappa(t)$ is the transmission function. On the time scale of the recrossings, about a picosecond, the transmission function decays from unity at $t=0$ to the so-called plateau level. The true rate constant is found by inserting this plateau value into Eq. (1.4). Actually, the plateau is not constant but decaying on the time scale of the reaction, but for most reactions this is too slow to be seen in MD simulations. In our previous article ${ }^{5}$ we discussed the calculation of the transmission function of the reaction studied here.

From Eqs. (1.1) and (1.3) one could infer that the calculated rate depends on the definition of the transition state, i.e., on $\xi^{\neq}$and $\xi\left(\left\{\mathbf{x}_{i}\right\}\right.$ ) (for simplicity we will neglect the latter dependence). The rate should of course depend on this definition, since it is embodied in the definition of the number of reactant molecules, $P_{R}$, and hence in the therefrom derived rate. But, provided the free-energy barrier is high and $\xi^{\neq}$lies somewhere near the top, the number of reactants is virtually independent to the precise location of $\xi^{\neq}$, and so must the rate be. The TST rate obviously strongly depends on $\xi^{\neq}$; in the RF method the dynamics incorporated in the transmission function blurs the picture. Miller ${ }^{6}$ showed, using Liouville's theorem, that the RF rate is independent of the precise location of the transition state. Chandler ${ }^{4}$ arrived at the same conclusion in a discussion based on the separation in time scales between recrossing and reactive events. In $\mathrm{Sec}$. III C this independence is verified numerically.

\section{THEORY}

\section{A. Reaction coordinate}

We here briefly discuss the unstable normal-mode reaction coordinate; an elaborate introduction is to be found in our previous article. ${ }^{5}$ The reasoning behind this reaction coordinate is the fact that in a reactive system most reactant to product trajectories will surmount the potential energy barrier somewhere near the lowest point of the barrier, i.e., the saddle point. It is therefore natural to construct a reaction coordinate based on the properties of the saddle point. The Taylor expansion of the potential energy around a saddle point, $\mathbf{X}^{0}$, up to second order reads

$$
\Phi(\mathbf{X})=\Phi\left(\mathbf{X}^{0}\right)+\frac{1}{2}\left(\mathbf{X}-\mathbf{X}^{0}\right)^{T} \mathbf{H}\left(\mathbf{X}-\mathbf{X}^{0}\right) .
$$

For notational convenience, we collect all coordinates of the reacting molecule in a single $3 N$-dimensional column vector of mass-weighted atomic coordinates, $\mathbf{X}^{T}=\left(\sqrt{m_{1}} \mathbf{x}_{1}^{T}\right.$, $\left.\sqrt{m_{2}} \mathbf{x}_{2}^{T}, \ldots, \sqrt{m_{N}} \mathbf{x}_{N}^{T}\right)$. The Hessian matrix $\mathbf{H}$ contains all second-order derivatives of the potential energy with respect to the components of $\mathbf{X}$. Its eigenvectors are orthogonal, or orthogonalizable in case of degeneracy, since the Hessian is symmetric. Henceforth all eigenvectors will be assumed to be normalized. We shall assume that there is no external field.

The set of $3 N$ eigenvectors can be split into three groups. First, there are three independent vectors $\mathbf{E}^{l}$ that correspond to an overall translation of the molecule. Obviously, they leave the potential energy unchanged and therefore are eigenvectors of $\mathbf{H}$ with eigenvalues zero, as follows from Eq. (2.1) after replacing $\mathbf{X}-\mathbf{X}^{0}$ by $\mathbf{E}^{l}$. Similarly, there are three independent (assuming a nonlinear molecule) eigenvectors $\mathbf{S}^{k}$ corresponding to infinitesimal rotations of the molecule. If $\mathbf{r}_{i}^{0}=\mathbf{x}_{i}^{0}-\mathbf{x}_{\mathrm{com}}^{0}$ is the vector pointing from the center of mass of the molecule to atom $i$, then an infinitesimal rotation around an axes $\mathbf{e}_{k}$ through the center of mass leads to atomic displacements

$$
\left(\mathbf{S}^{k}\right)^{T}=\left(\sqrt{m_{1}}\left(\mathbf{e}_{k} \times \mathbf{r}_{1}^{0}\right)^{T}, \sqrt{m_{2}}\left(\mathbf{e}_{k} \times \mathbf{r}_{2}^{0}\right)^{T}, \ldots, \sqrt{m_{N}}\left(\mathbf{e}_{k} \times \mathbf{r}_{N}^{0}\right)^{T}\right) .
$$

Obviously, assuming orthonormality of the eigenvectors $\mathbf{S}^{k}$, the three $\mathbf{e}_{k}$ are not orthogonal. The six modes of the first two groups are degenerate, i.e., they all have eigenvalue zero, if there is no external field. Finally, the remaining $3 N-6$ eigenvectors $\mathbf{Q}^{j}$ are the normal modes of vibration.

At an energy minimum all vibrational modes have a nonnegative eigenvalue, but at a first-order saddle point there will be exactly one mode, $\mathbf{Q}^{r}$, with a negative eigenvalue. It is this unstable normal mode that we are interested in; it points downhill, from the saddle point towards the reactant and product wells, while all other modes are pointing uphill. Motion of the molecule along this mode, therefore, corresponds to a reaction. The reaction coordinate of a molecule with coordinates $\mathbf{X}$ might now be defined as the projection of the displacement with respect to the saddle-point configuration onto the unstable normal mode,

$$
\xi=\left(\mathbf{X}-\mathbf{X}^{0}\right) \cdot \mathbf{Q}^{r} .
$$

This coordinate, however, is not invariant under rotations of the molecule $\mathbf{X}$. This problem is solved by making the saddle point $\mathbf{X}^{0}$ depend on $\mathbf{X}$. First we note that the rotated saddle point $\mathbf{A} \mathbf{R}^{0}$, where $\mathbf{A}$ is a $3 N$-dimensional rotation matrix containing $N$ copies of a regular three-dimensional rotation matrix $\mathbf{a}$ down the diagonal and $\mathbf{R}^{0}$ is a mass-weighted column vector of the $\mathbf{r}_{i}^{0}$, is also a saddle point. The normal modes of this rotated configuration simply are $\mathbf{E}^{l}, \mathbf{A} \mathbf{S}^{k}$, and $\mathbf{A} \mathbf{Q}^{j}$. We next define the reaction coordinate by

$$
\xi=\left(\mathbf{X}-\mathbf{A} \mathbf{R}^{0}\right) \cdot \mathbf{A} \mathbf{Q}^{r} .
$$

The rotation matrix $\mathbf{A}$, which is a function of just three parameters, is determined from the three equations

$$
0=\left(\mathbf{X}-\mathbf{A} \mathbf{R}^{0}\right) \cdot \mathbf{A} \mathbf{S}^{k}, \quad k \in\{1,2,3\},
$$

and assures rotational invariance. One may check that the above two equations are invariant under translation, as follows from the orthogonality of the eigenvectors of the Hessian.

The inverse transformation reads

$$
\mathbf{X}=\mathbf{A} \mathbf{R}+\sum_{l=1}^{3} \gamma_{l} \mathbf{E}^{l}, \quad \mathbf{R}=\mathbf{R}^{0}+\sum_{j=1}^{3 N-6} \alpha_{j} \mathbf{Q}^{j},
$$

where the center of mass of $\mathbf{R}$ evidently lies at the origin. The reaction coordinate is one of the deviations, $\xi=\alpha_{r}$. At this point the reader might argue that the reaction coordinate now is dimensionless, while in Eq. (2.4) it seems to have a 
dimension of $\mathrm{kg} \mathrm{m}^{2}$. However, the orthonormality of the vectors $\mathbf{Q}^{j}$ that is assumed in going from Eq. (2.6) to Eq. (2.4) cancels the dimensionality.

In the following we will make use of the derivative of $\xi$ with respect to $\mathbf{X}$. From the orthogonality of the rotation matrix in combination with the derivatives of Eqs. (2.4) and (2.5) it follows that

$$
\nabla_{X} \xi=\mathbf{A}\left(\mathbf{Q}^{r}-\sum_{k=1}^{3} d_{k}^{r} \mathbf{S}^{k}\right) .
$$

The expression relating $d_{k}^{r}$ to $\mathbf{X}, \mathbf{A}, \mathbf{S}^{k}$, and $\mathbf{Q}^{r}$ is given in our previous article. ${ }^{5}$

\section{B. Theory of small vibrations}

The free energy of a classical molecule in vacuo can be calculated in reasonable approximation by the theory of small vibrations. If we assume that the potential energy function around the energy minimum is quadratic, and if we neglect the coupling between rotations and vibrations, then the well-known semiclassical partition function of the minimum reads $^{7}$

$$
\begin{aligned}
Q= & \frac{1}{h^{3 N}} \int d \mathbf{x} \int d \mathbf{p}_{x} e^{-\beta H\left(\mathbf{x}, \mathbf{p}_{x}\right)} \\
= & \left(\frac{2 \pi M k T}{h^{2}}\right)^{3 / 2} V \cdot \frac{\pi^{1 / 2}}{\sigma}\left(\frac{8 \pi^{2} k T}{h^{2}}\right)^{3 / 2}\left(I_{A} I_{B} I_{C}\right)^{1 / 2} \\
& \times \prod_{i=1}^{3 N-6} \frac{k T}{\hbar \omega_{i}} \cdot \omega_{e} e^{-E_{\min } / k T} .
\end{aligned}
$$

Here $\beta=1 / k T, T$ is the absolute temperature, $k$ is the Boltzmann constant, $H$ is the Hamiltonian, $h$ is the Planck constant, $\hbar=h / 2 \pi, M$ is the total mass of the molecule, $V$ is the volume of the box, $\sigma$ is the symmetry number, the $I_{X}$ are the momenta of inertia, the $\omega_{i}$ are the eigenfrequencies of vibration (the square roots of the nonzero eigenvalues of the Hessian), $\omega_{e}$ is the electronic degeneracy, and $E_{\min }$ is the energy at the potential minimum. We will now give a derivation of this expression, such that it later can be generalized to calculate a $\xi$-dependent partition function.

To solve the integral of Eq. (2.8) we use the generalized coordinates of Eq. (2.6), where now the configuration $\mathbf{R}^{0}$ is assumed to be a local minimum of the potential function, and $\mathbf{S}^{k}, \mathbf{E}^{l}$, and $\mathbf{Q}^{j}$ are evaluated at this point. The obvious reason is that the potential energy in terms of these coordinates reduces to a simple formula, $\Phi=E_{\min }+\frac{1}{2} \Sigma_{j=1}^{3 N-6} \omega_{j}^{2} \alpha_{j}^{2}$, provided the vibrations are small. After the canonical transformation of Eq. (2.8) from Cartesian coordinates to massweighted Cartesian coordinates the integration over the conjugate momenta yields $(2 \pi k T)^{3 N / 2}$. The subsequent transformation of the coordinates to the generalized coordinates $\psi_{k}, \alpha_{j}$, and $\gamma_{l}$ is accompanied by the Jacobi matrix

$$
\mathbf{J}=\frac{\partial \mathbf{X}}{\partial\left\{\psi_{k}, \alpha_{j}, \gamma_{l}\right\}}=\mathbf{A}\left(\begin{array}{lll}
\Psi^{k} & \mathbf{Q}^{j} & \mathbf{A}^{-1} \mathbf{E}^{l}
\end{array}\right),
$$

where the matrix is expressed in terms of $3+(3 N-6)+3$ column vectors, and the three $\psi_{k}$ parametrize the rotation matrix A. Since the derivative of the rotation matrix with respect to its $k$ th argument can be written as the product of the rotation matrix and an antisymmetric matrix $\mathbf{B}_{k}$ we find

$$
\Psi^{k}=\mathbf{B}_{k}(\psi) \mathbf{R}=\sum_{l=1}^{3} \epsilon^{l} \mathbf{R} c_{l k}(\psi) .
$$

Here the $\epsilon^{l}$ are three $3 N$-dimensional matrices containing $N$ copies of a three-dimensional antisymmetric matrix, an infinitesimal rotation generator, ${ }^{8}$ down the diagonal. The Jacobi matrix can then be written as

$$
\mathbf{J}=\mathbf{A}\left(\begin{array}{lll}
\epsilon^{k} \mathbf{R} & \mathbf{Q}^{j} & \mathbf{A}^{-1} \mathbf{E}^{l}
\end{array}\right)\left(\begin{array}{ccc}
\mathbf{c} & \mathbf{0} & \mathbf{0} \\
\mathbf{0} & \mathbf{1} & \mathbf{0} \\
\mathbf{0} & \mathbf{0} & \mathbf{1}
\end{array}\right) .
$$

If the Euler angles ${ }^{8}$ are used to parametrize the rotation matrix, then $0 \leqslant \psi_{1} \leqslant 2 \pi, 0 \leqslant \psi_{2} \leqslant \pi, 0 \leqslant \psi_{3} \leqslant 2 \pi$, and $|\mathbf{c}|$ $=\sin \psi_{2}$. Obviously, $|\mathbf{A}|=|\mathbf{a}|^{N}=1$.

For small vibrations we may approximate $\mathbf{R}$ by $\mathbf{R}^{0}$ in Eq. (2.11). Then $\epsilon^{k} \mathbf{R}$ is of the form of Eq. (2.2), hence perpendicular to both $\mathbf{A}^{-1} \mathbf{E}^{l}$ and $\mathbf{Q}^{j}$. Denoting the second matrix on the right-hand side of Eq. (2.11) by $\mathbf{m}$, and using $|\mathbf{m}|=\left|\mathbf{m}^{T} \mathbf{m}\right|^{1 / 2}$ in combination with these orthogonality relations and the orthonormality of the eigenvectors of the Hessian, the resulting Jacobian reads

$$
|\mathbf{J}|=\left|\mathbf{I}^{0}\right|^{1 / 2} \sin \psi_{2}=\left(I_{A}^{0} I_{B}^{0} I_{C}^{0}\right)^{1 / 2} \sin \psi_{2} .
$$

The inertia tensor $\mathbf{I}^{0}$ is the upper left $3 \times 3$ submatrix of $\mathbf{m}^{T} \mathbf{m}$, with elements $I_{l k}^{0}=\left(\epsilon^{l} \mathbf{R}^{0}\right)^{T}\left(\epsilon^{k} \mathbf{R}^{0}\right)$, and the $I_{X}^{0}$ are the eigenvalues of this tensor. The integrals over the generalized coordinates are straightforward, and combination with the previously evaluated integral over the momenta leads to Eq. (2.8). One should keep in mind that in a cubic box of side $L$ the coordinates $\gamma_{l}$ run from 0 to $L \sqrt{M}$ due to the normalization of the translational eigenvectors. The integrals over the $\alpha_{j}$ are supposed to run from minus infinity to plus infinity.

In order to be able to calculate the numerator in the second factor in Eq. (1.2), we now derive an expression for the partition function as a function of the reaction coordinate,

$$
Q\left(\xi^{*}\right)=\frac{1}{h^{3 N}} \int d \mathbf{x} \int d \mathbf{p}_{x} e^{-\beta H\left(\mathbf{x}, \mathbf{p}_{x}\right)} \delta\left[\xi(\mathbf{x})-\xi^{*}\right],
$$

using the same assumptions that were used in the above derivation of Eq. (2.8). This partition function is often expressed in terms of a free energy by

$$
A\left(\xi^{*}\right)=-k T \ln Q\left(\xi^{*}\right) .
$$

We first transform to mass-weighted coordinates, and define a standard minimum $\mathbf{R}^{*}$ on the hyperplane $\xi(\mathbf{X})=\xi^{*}$. This minimum is found by varying the $\alpha_{j}$ in Eq. (2.6), while keeping $\alpha_{r}$ equal to $\xi^{*}$. The minimum $\mathbf{R}^{*}$ is not rotated with respect to $\mathbf{R}^{0}$, and their centers of mass coincide. In the present partition function the configuration $\mathbf{R}^{*}$ plays a role analogous to that of $\mathbf{R}^{0}$ in Eq. (2.8). In the Appendix it is shown that 


$$
\begin{aligned}
Q\left(\xi^{*}\right)= & \left(\frac{2 \pi M k T}{h^{2}}\right)^{3 / 2} V \cdot \frac{\pi^{1 / 2}}{\sigma}\left(\frac{8 \pi^{2} k T}{h^{2}}\right)^{3 / 2}\left(I_{A}^{*} I_{B}^{*} I_{C}^{*}\right)^{1 / 2} \\
& \times \prod_{i=1}^{3 N-7} \frac{k T}{\hbar \omega_{i}^{*}} \cdot \omega_{e} e^{-E^{*} / k T} \cdot\left|\nabla_{\mathbf{R}^{*}} \xi\right|^{-1} \\
& \cdot(2 \pi k T)^{1 / 2} h^{-1},
\end{aligned}
$$

where a superscript asterisk denotes a quantity that is to be calculated at $\mathbf{R}^{*}$. An alternative expression for the partition function is also derived in the Appendix.

To locate the minimum $\mathbf{R}^{*}$ on the hyperplane $\xi(\mathbf{X})$ $=\xi^{*}$ we used a standard minimum search routine in combination with a constraint on the reaction coordinate. The resulting configuration was further refined by reducing the projection of the gradient of the potential onto the tangent hyperplane. This was achieved by an iterative NewtonRaphson zero-point search, using forces and Hessians calculated in the plane spanned by the basis vectors $\mathbf{T}^{l}$ defined in the Appendix. Since the tangent hyperplane is only locally correct, each iterated point was reset to $\xi(\mathbf{X})=\xi^{*}$ by a SHAKE routine, ${ }^{5,9}$ followed by a recalculation of the normal to the hyperplane, $\mathbf{N}$, and of the basis vectors $\mathbf{T}^{l}$. If these minimizations are performed in the full $3 N$-dimensional space, then the molecule will rotate inevitably. This is easily corrected for, either by rotating the vectors $\mathbf{N}$ and $\mathbf{T}^{l}$, or by counterrotating the molecule after each step. This effect does not arise if the minimum is directly located in the $3 N-6$ dimensional $\alpha$ space.

Equations (2.8) and (2.15) obviously do not take into account the anharmonicity of the force field, nor the coupling between rotations and vibrations. The inclusion of interactions with a solvent is difficult. In the next section an alternative technique is discussed which does not suffer from these drawbacks.

\section{Umbrella sampling}

A straightforward way of obtaining the correct probability distribution $P(\xi)$ of a (dissolved) molecule is to calculate this distribution directly from a long MD run. The MD run should include numerous barrier crossings to ensure that both wells and the barrier region are sampled sufficiently. For reactions with a high barrier, exceeding several $k T$, such a run would last prohibitively long. Two techniques are commonly employed to circumvent this problem. ${ }^{10-12}$ In the potential of mean force method the derivative of the free energy with respect to the reaction coordinate is calculated in a series of $\xi$-constrained runs and then integrated. We will use the second method and apply an "umbrella potential," a modification to the force field that can easily be corrected for, to effectively reduce the barrier.

We are interested in the probability distribution of the reaction coordinate,

$$
P\left(\xi^{*}\right)=\int d \mathbf{X} p(\mathbf{X}) \delta\left[\xi(\mathbf{X})-\xi^{*}\right]
$$

Here

$$
p(\mathbf{X})=\frac{1}{Z} e^{-\beta \Phi(\mathbf{X})}
$$

is the Boltzmann factor for a molecule in configuration $\mathbf{X}$ with potential $\Phi$, and $Z$ is a normalization factor. $\mathbf{X}$ represents all coordinates, including the solvent coordinates, and $\Phi$ includes solvent-solvent and solute-solvent interactions. In the barrier region this probability becomes extremely small, too small to be sampled efficiently. Addition of an umbrella potential, $U$, changes the Boltzmann factor into

$$
p_{U}(\mathbf{X})=\frac{1}{Z_{U}} e^{-\beta[\Phi(\mathbf{X})+U(\mathbf{X})]} .
$$

The umbrella potential is used to favor the distribution $p_{U}$ in areas that would otherwise have been difficult to sample. The requested probability distribution of the reaction coordinate is readily obtained from the biased distribution by combining Eqs. (2.16) through (2.18) into

$$
P\left(\xi^{*}\right)=\frac{Z_{U}}{Z} \int d \mathbf{X} p_{U}(\mathbf{X}) e^{\beta U(\mathbf{X})} \delta\left[\xi(\mathbf{X})-\xi^{*}\right] .
$$

The normalization factors $Z_{U}$ and $Z$ do not have to be calculated independently, only the factor which normalizes the final distribution $P\left(\xi^{*}\right)$ is needed.

In many applications the umbrella potential is chosen to be a function of the reaction coordinate $\xi$ only, in which case the exponential in the integral of Eq. (2.19) becomes a constant. $^{13,14}$ For molecules with more than two local minima, as in our case, it may prove necessary to use a somewhat more complicated umbrella potential to suppress unwanted side reactions.

Often a series of $L$ umbrellas of the form $\Phi_{l}(\xi)=\frac{1}{2} k(\xi$ $\left.-\xi_{l}\right)^{2}, l=1, \ldots, L$, is used. At each $l$ a small area of configuration space, a so-called "window," is sampled, resulting in a distribution $P_{l}(\xi)$ for each window. The various distributions are then combined into a single distribution, using the overlap of successive windows to match the partial distributions. ${ }^{15}$ We use a single umbrella covering the whole range of $\xi$. Only if the diffusion along the reaction coordinate is relatively fast will this method work, so care must be taken to make the reaction coordinate distribution in the biased run as flat as possible.

\section{Transition state crossing velocity}

The first term on the right-hand side of Eq. (1.2) is most easily calculated by using the mass-weighted coordinates introduced above. The velocity of the reaction coordinate can be expressed in terms of the mass-weighted Cartesian momenta as

$$
\dot{\xi}=\nabla_{X} \xi \cdot \dot{\mathbf{X}}=\nabla_{X} \xi \cdot \mathbf{p}_{X},
$$

and the kinetic energy of the molecule reduces to $E_{\text {kin }}$ $=\frac{1}{2} \mathbf{p}_{X} \cdot \mathbf{p}_{X}$. These equations remain the same if the coordinate system is rotated. We now apply a rotation such that the first mass-weighted coordinate axis lies parallel to $\nabla_{X} \xi$; all other axes are then perpendicular to the gradient. Integration over the momentum conjugate to this axis, using 


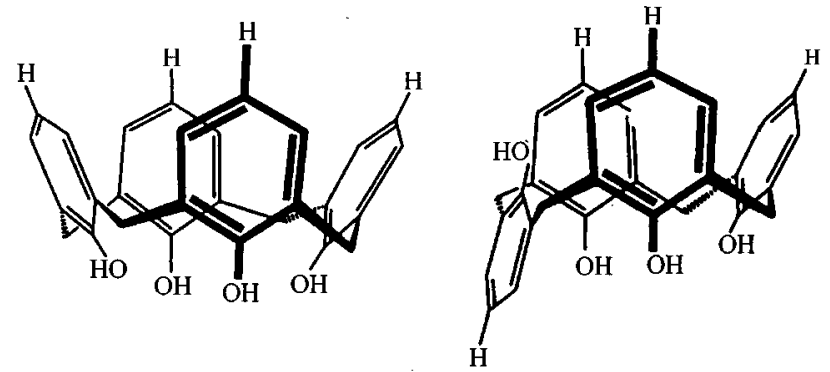

FIG. 1. The cone (left) and partial cone (paco, right) conformation of a calix[4]arene.

$$
\int_{0}^{\infty} d p p e^{-a p^{2}}=\frac{1}{2 \sqrt{\pi a}} \int_{-\infty}^{\infty} d p e^{-a p^{2}}
$$

yields the average positive crossing velocity

$\frac{\left\langle\delta\left[\xi(0)-\xi^{\ddagger}\right] \dot{\xi}(0) \theta[\dot{\xi}(0)]\right\rangle}{\left\langle\delta\left[\xi(0)-\xi^{\ddagger}\right]\right\rangle}=\sqrt{\frac{k T}{2 \pi}} \frac{\left\langle\delta\left[\xi(0)-\xi^{\ddagger}\right]\left|\nabla_{X} \xi\right|\right\rangle}{\left\langle\delta\left[\xi(0)-\xi^{\ddagger}\right]\right\rangle}$.

The length of the gradient, Eq. (A8), is seen to act as the reciproke effective mass of the reaction coordinate. Combining Eqs. (1.2), (2.8), (2.15), and (2.22) yields the classical analogue to Eyrings expression.

It is common practice to express the rate constant in terms of a free-energy difference between the transition state and the reactant well, $\Delta A^{\neq}=A^{\neq}-A_{R}$, as in

$$
k_{f}=\frac{k T}{h} e^{-\Delta A^{\neq} / k T} .
$$

This free-energy difference should not be confused with the free-energy difference calculated by means of Eqs. (1.2) and (2.14). First, the free energy $A^{\neq}$is based on the partition function of a molecule which is constrained to $\xi(\mathbf{X})=\xi^{\neq}$ and which explicitly excludes any motion along the reaction coordinate, $\dot{\xi}=0$. In the harmonic approximation

$$
A^{\neq}=-k T \ln \left[Q\left(\xi^{\neq}\right) \frac{h}{\sqrt{2 \pi k T}}\right] .
$$

Using this result, the transition state theory value of the rate constant in the harmonic approximation takes the form of Eq. (2.23). Second, in the literature on experiments the $\Delta A^{\neq}$ includes the transmission factor.

\section{RESULTS}

The techniques introduced above are now applied to the isomerization reaction of calix[4]arene. ${ }^{16}$ This molecule consists of four phenol groups, each of which is connected to two neighboring phenols by methyl bridges located ortho to the hydroxyl group, see Fig. 1. In supramolecular chemistry they are being used as building blocks. The interesting property in the current context is the fact that they have four stable conformations. The "cone" conformation, with all phenol groups pointing in the same direction, is most abundant. It is stabilized by a cyclic array of four hydrogen bonds. The partial cone, "paco" for short, is formed by rotating one phenol group, with the methyl groups acting as hinges and the hydroxyl group moving through the central annulus. In this process two hydrogen bonds are broken. We will focus here on this reaction step. In a following reaction step one of the phenol groups nearest to the rotated phenol can rotate and form the "1,2-alternate," or the opposing phenol group can rotate to form the "1,3-alternate." After two more steps all phenol groups have rotated, and the final "inverted cone'" conformation is reached. This name will be used to distinguish it from the initial "cone" configuration.

All simulations were done with a modified version of GROMOS87. ${ }^{17}$ Several routines were adapted or added for the handling of the normal-mode reaction coordinate. The calix[4]arene was modeled with the all-atom CHARMM parameter set $22 .^{18}$ The saddle point of the cone to paco reaction was calculated with the conjugate peak refinement algorithm. ${ }^{18,19}$ The unstable normal mode of the saddle point, $\mathbf{Q}^{r}$, was chosen to point towards the paco well. All normal modes were normalized to $1 \mathrm{amu}^{1 / 2} \mathrm{~nm}$. In the MD runs all bonds involving hydrogen atoms were constrained to constant lengths using SHAKE. ${ }^{9}$ The effect of this constraint on the sampling of phase space was shown to be very small, so we applied no corrections. We refer to our previous article ${ }^{5}$ for more details.

In Sec. III A the free-energy profile of a calix[4]arene in vacuo is calculated using the theory of small vibrations. The runs with umbrella sampling, both in vacuo and in chloroform, are described in Sec. III B. Combining the free energy and the transmission function allows us to calculate the reaction rate of Sec. III C.

\section{A. Small vibrations}

The minimum energy of a calix[4]arene in vacuo as a function of the reaction coordinate was calculated by two series of $\xi$-constrained energy minimizations. Starting at the saddle point, the value of $\xi$ was increased (decreased) by 0.01 up to a maximum value of $2(-2)$, and a minimum was located at each value. The resulting function is shown in Fig. 2 by the dotted line, while the plot is shifted vertically to place the saddle point at zero energy. The local minima of the cone and paco conformations are seen to be at 15.2 and $5.6 \mathrm{kcal} / \mathrm{mol}$, respectively, below the saddle point, in agreement with the results of previous unconstrained minimization runs. ${ }^{18}$ At both extremes of the $\xi$ axis, with energies exceeding the saddle-point value, the minimum search algorithm ran into problems. In these areas, as a result of the constraint on the reaction coordinate, the strain on the molecules was so extreme that the molecule "spontaneously" changed into another conformation. These areas are irrelevant to the problem at hand, so these problems are of no consequence. For energies below the saddle-point value, in the range $-1.71 \leqslant \xi$ $\leqslant 1.53$, the minimization produces a smooth energy profile.

Next, each minimum in the intermediate range was refined by a Newton-Raphson zero-point search in $3 N$ dimensions, as described in Sec. II B. After each iteration step SHAKE was applied to prevent the molecule from slowly drifting away from the hyperplane. The Hessian $\mathbf{H}^{*}$, as de- 


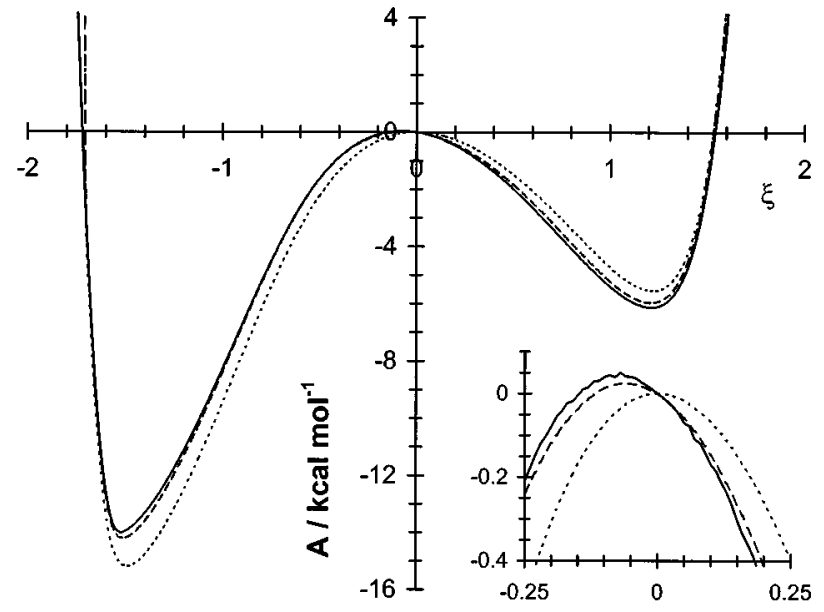

FIG. 2. Minimum energy (dotted), free energy according to normal-mode analysis (dashed), and free energy according to umbrella sampling (solid) for a calix[4]arene in vacuo. The inset shows the three functions near the saddlepoint.

fined in the Appendix, of the converged configurations were diagonalized to obtain the eigenfrequencies of vibration. All frequencies were positive, so Eq. (2.15) could be used to evaluate the partition function. The absolute values of the rotational eigenfrequencies, which should have been zero, were found to be smaller than the lowest vibrational eigenfrequency by 4 to 5 orders of magnitude for most configurations, indicating that the constrained minima were indeed well converged.

The free-energy function in vacuo was calculated by means of Eq. (2.15). In Fig. 2 this function is shown as a dashed line, shifted vertically in order to make it pass through the origin. The difference with the minimum energy function is considerable, ranging from $+1.2 \mathrm{kcal} / \mathrm{mol}$ near the cone minimum to $-0.5 \mathrm{kcal} / \mathrm{mol}$ near the paco minimum. This difference is predominantly of vibrational origin, i.e., it reflects the $\xi$ dependence of the eigenfrequencies of vibration perpendicular to the gradient of $\xi$. The other two $\xi$-dependent terms, due to rotation and due to the gradient of $\xi$, vary by just $2.9 \%$ and $0.8 \%$, respectively, of the variation of the vibrational contribution. The top of the free-energy function has shifted from the origin towards $\xi=-0.06$, as can be seen in the inset of Fig. 2. The transmission function calculated in vacuo with $\xi^{\neq}=0$ must therefore yield a transmission coefficient less then unity, as was indeed found. ${ }^{5}$

In an alternative calculation the potential energy as a function of the $\alpha_{j}$, Eq. (2.6), was minimized using NewtonRaphson, at intervals of 0.01 for $\xi$. The partition function was then calculated according to Eq. (A13) of the Appendix. The resulting minimum energy and free-energy distributions are identical to the ones discussed above, but the two methods are clearly different in the assignment of the rotational and vibrational free energy.

Replacing the vibrational partition function by its quantized version is of little effect on the free-energy function: after forcing the function to pass through the origin, the differences with the semiclassical result range from about
$+0.25 \mathrm{kcal} / \mathrm{mol}$ for the cone to $-0.2 \mathrm{kcal} / \mathrm{mol}$ for the paco. However, now the two expressions for the partition function differ by at most $0.02 \mathrm{kcal} / \mathrm{mol}$. Omission of the 24 highest frequencies, i.e., those resulting from the hydrogen stretching modes which will be constrained in the MD runs, is of little influence, namely of the order of $0.06 \mathrm{kcal} / \mathrm{mol}$ for the quantized partition function and $0.008 \mathrm{kcal} / \mathrm{mol}$ for the semiclassical partition function.

\section{B. Umbrella sampling}

As the initial trial umbrella for the umbrella sampling in vacuo served the above calculated minimum energy function, ${ }^{13} U(\mathbf{X})=f(\xi)$. At first a tenth-order polynomial was used to fit the function in the interval where $\xi$ ranges from -1.7 to +1.5 , i.e., the region in which the potential energy is less than the saddle-point energy. The umbrella was supplemented with two Fermi-Dirac functions at both extremes to delimit the range of accessible $\xi$ values to those values that are relevant to the isomerization rate. The leastsquares fitted polynomial oscillated around the minimum energy function with an amplitude of about $0.25 \mathrm{kcal} / \mathrm{mol}$. These oscillations were clearly visible in the sampled probability distribution, which contained corresponding maxima and minima. Upon examination of the trajectory it became clear that the molecule was hopping from one local minimum to the next. Since the time spent in the evaluation of the umbrella potential is negligible in comparison with the overall execution time, a more complicate umbrella, which represented the minimum energy function better, was used next. The cone well and the paco well were both fitted with a fifteenth-order polynomial. In the saddle-point region, which was covered by both fits, a third-order "switch" polynomial was used to make a smooth transition from one fit to the other. With this umbrella the time evolution of the reaction coordinate was effectively smoothed.

A second problem arose during the simulations. When the reaction coordinate reached a value of about 0.7 , it proved possible for a phenyl ring neighboring the freely rotating ring to flip over. Thus the calix[4]arene reached the 1,2-alternate configuration, where it was captured for the rest of the MD run. The reason for this problem was that the umbrella not only lowers the energies of the paco and the barrier conformations to the energy of the cone conformations, but it also lowers other saddle points to within a few $k T$ of the cone conformations. We therefore located the saddle point of the paco to 1,2-alternate transition, in the absence of an umbrella, using the TRAVEL algorithm and refined it with Newton-Raphson. Because of the circular orientation of the hydrogen bonds at the lower rim of the calix[4]arene there are two saddle points, depending on which neighboring phenyl ring is being rotated. One saddle point is located at $\xi=1.02$ with an energy of $0.5 \mathrm{kcal} / \mathrm{mol}$ below the saddle point of the cone to paco transition, the other saddle point is located at $\xi=0.96$ with an energy of 0.3 $\mathrm{kcal} / \mathrm{mol}$ above the saddle point of the cone to paco transition. This second saddle point was not mentioned by Fischer et al. ${ }^{18}$ The umbrella potential was next extended to

$$
U(\mathbf{X})=f(\xi)+f_{1}\left(\xi_{1}\right)+f_{2}\left(\xi_{2}\right),
$$




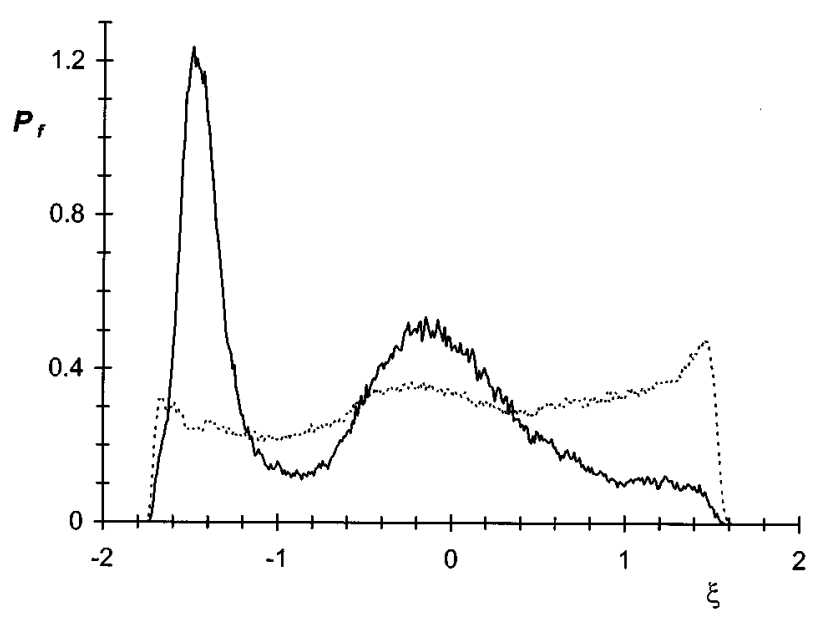

FIG. 3. Probability distributions $P_{f}(\xi)$ in vacuo (dotted) and in chloroform (solid), calculated with the same umbrella potential. The areas under both graphs are identical.

where $f_{1}$ and $f_{2}$ are two Fermi-Dirac functions used to delimit the range of accessible values of the reaction coordinates $\xi_{1}$ and $\xi_{2}$ calculated with respect to the saddle points of the paco to 1,2-alternate transitions. Analysis of the configurations sampled in simulations showed that these two reaction coordinates are well behaved and of constant sign throughout the cone and paco wells. Inclusion of the two potentials hardly effected the motion of the molecule for most of the time, but it effectively suppressed the rare but fatal transitions to the 1,2 alternate. Of course, the two additional potential functions were corrected for in the evaluation of the probability distribution of $\xi$, see Eq. (2.19).

A calix[4]arene in vacuo with the above described umbrella potential was equilibrated at a temperature of $300 \mathrm{~K}$ using velocity scaling. When a steady temperature was reached the velocity scaling was turned off, the angular velocity was eliminated and the molecule was simulated for 30 ns. The resulting biased probability distribution $P_{f}(\xi)$, corrected for $f_{1}$ and $f_{2}$ but not for $f$, contained some strongly preferred regions. The vast majority of the sampled configurations was distributed over the paco well, and a peak was found at the outer extreme of the cone well. The cone well itself, i.e., the region with the highest population in the unbiased distribution, was sampled very poorly. To improve the sampling of this region, the probability distribution was transformed into a potential which was added to the existing umbrella. A second $30 \mathrm{~ns}$ run and a repeat of this recipe eventually lead to the relatively flat distribution $P_{f}(\xi)$ of Fig. 3 . The resulting free-energy profile after correction for $f$ is shown in Fig. 2 as a solid line. Note the excellent resemblance to the normal-mode based free-energy function, they differ by less than $0.3 \mathrm{kcal} / \mathrm{mol}$, and the less striking resemblance to the minimum energy function.

The final vacuum umbrella was also used in a run of a calix[4]arene dissolved in chloroform $\left(\mathrm{CHCl}_{3}\right)$. The run was performed at a constant temperature of $300 \mathrm{~K}$ and a constant pressure of $1 \mathrm{bar}$, bonds containing hydrogen atoms were constrained, the time step was 2 fs, long-range interactions were cut off beyond $1.3 \mathrm{~nm}$, and the periodic box was a truncated octahedron containing 324 rigid solvent molecules. The system was equilibrated for $150 \mathrm{ps,} \mathrm{followed} \mathrm{by} \mathrm{a} \mathrm{pro-}$ duction run of $2 \mathrm{~ns}$. The sampled probability distribution $P_{f}(\xi)$ is shown in Fig. 3 as a solid line. Comparison with the vacuum distribution shows that the solvent environment induces a preference for the cone and the saddle-point configurations. Fortunately, the differences with the vacuum run are fairly small since otherwise it would have been impossible to make the leap from vacuum to solvent without breaking up the range of the umbrella into smaller parts. Once more, the probability distribution was turned into a potential and added to the umbrella. This umbrella was then used in a $5 \mathrm{~ns}$ simulation to sample the conclusive distribution of the reaction coordinate of the dissolved molecule.

\section{Rate constants}

In this section the free-energy profiles of the previous two sections are used to calculate rate constants according to transition state theory. The transmission coefficient is calculated next, and the reactive flux rate is obtained by multiplication of this coefficient with the transition state theory rate constant.

We first discuss the isomerization rate of a calix[4]arene in vacuo. The partition function of the cone well, $Q_{\text {cone }}$, is equal to the integral of the partition function $Q(\xi)$, Eq. (2.13), over the interval from minus infinity to $\xi^{\neq}$. Because of the depth of the well the result is virtually independent of $\xi^{\neq}$. With $\xi^{\neq}=0$ and the approximation of small vibrations we find $A_{\text {cone }}=172.7 \mathrm{kcal} / \mathrm{mol}$, and similarly, $A_{\text {paco }}=180.6$ $\mathrm{kcal} / \mathrm{mol}$. Direct evaluation of the free energies with the full normal-mode analysis of Eq. (2.8) yields almost identical values. Next, we look at the transition state theory rate constant as a function of $\xi^{\neq}$. Approximating $|\nabla \xi|=\left|\mathbf{Q}^{r}\right|$, which is exact at the saddle point, we find that transition state theory predicts a minimum rate constant of $167 \mathrm{~s}^{-1}$ at $\xi$ $=-0.06$, corresponding to the maximum of the free energy in Fig. 2. If, following Eyring, we use $\xi^{\neq}=0$ as the transition state, we find $A(O)=185.9 \mathrm{kcal} / \mathrm{mol}$ and $k_{f}^{\mathrm{TST}}$ $=174 \mathrm{~s}^{-1}$. Using the free-energy $A_{\text {cone }}$ obtained with Eq. (2.8) in this case, i.e., using the textbook equation in which the rate constant is expressed in terms of the energies and the eigenfrequencies of the reactant well and the saddle point, ${ }^{2,20}$ we find a value of $177 \mathrm{~s}^{-1}$.

The changes of the free-energy profile in going from the normal-mode analysis to the umbrella sampling method are found to be fairly small. Nevertheless, the population of the paco well nearly doubles from $1.8 \times 10^{-6}$ to $3.4 \times 10^{-6}$, while $A_{\text {paco }}-A_{\text {cone }}$ changes from 7.9 to $7.5 \mathrm{kcal} / \mathrm{mol}$. At $\xi$ $=0$ the population rises by about $50 \%$, while $A(O)-A_{\text {cone }}$ changes from 13.1 to $12.9 \mathrm{kcal} / \mathrm{mol}$, and the rate rises to $k_{f}^{\mathrm{TST}}=256 \mathrm{~s}^{-1}$. Combination with the previously established transmission coefficient ${ }^{5}$ of 0.923 at $t=0.6$ ps yields the reactive flux method rate of $237 \mathrm{~s}^{-1}$. The transmission coefficient after 2 ps is slightly lower, $\kappa=0.82$, and leads to $k_{f}^{\mathrm{RF}}$ $=209 \mathrm{~s}^{-1}$.

In principle the rate constant should be independent of 


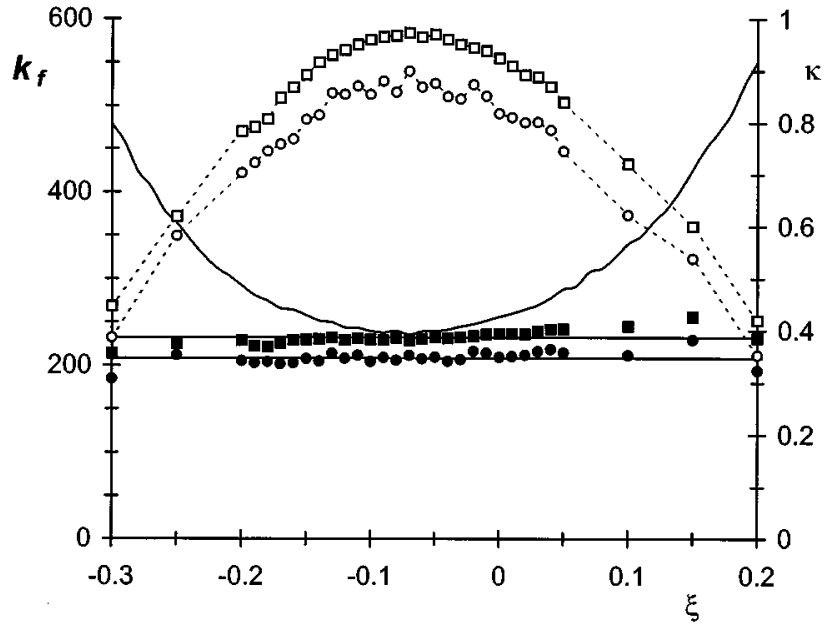

FIG. 4. Transition state theory rate (solid), transmission coefficient (open), and reactive flux rate (solid) as a function of the location of the transition state in vacuo, for $t=0.6 \mathrm{ps}$ (squares) and $t=2.0 \mathrm{ps}$ (circles).

the precise choice of the transition state, as long as this state is close enough to the top of the free-energy barrier. To test this requirement in the case of the reactive flux method and to verify our results, we have calculated the rate constant as a function of $\xi^{\neq}$. At various values the transmission function was evaluated by performing 2000 relaxation runs following the procedure outlined in our previous article. These transmission functions behaved as expected. For positive $\xi^{\neq}$virtually all molecules with a positive transient velocity, $\dot{\xi}(0)$, ended up as a paco. A considerable amount of those with a negative transient velocity also ended up as paco, and their number increased with increasing $\xi^{\neq}$. For negative $\xi^{\neq}$it was the other way around: many molecules with a positive transient velocity were found to recross the transition state, while molecules with a negative transient velocity recrossed only rarely. As reported earlier, a significant fraction of the molecules entering the paco well was found to return to the cone after a single oscillation of about $0.7 \mathrm{ps}$ in the paco well. We therefore calculated the reactive flux rate constant using two transmission coefficients, namely the value of $\kappa(t)$ at $0.6 \mathrm{ps}$, as if the wells are perfect sinks, and the one at 2.0 ps. In Fig. 4 it is evident that the rates hardly depend on the transition state, as it should be.

The effect of the chloroform solvent on the calix[4]arene is to shift the probability distribution to the cone conformation, leading to an equilibrium constant of $2.4 \times 10^{-7}$ and $A_{\text {paco }}-A_{\text {cone }}=9.1 \mathrm{kcal} / \mathrm{mol}$. With $A(O)-A_{\text {cone }}=13.6 \mathrm{kcal} /$ mol the transition state rate decreases to $84 \mathrm{~s}^{-1}$. The transmission function for this system ${ }^{5}$ was found to be 0.43 , so the reactive flux rate equals $36 \mathrm{~s}^{-1}$. The solvent is thus seen to reduce the reaction rate by a factor of 6 .

In our previous article we calculated the transmission coefficient from a set of 2000 relaxation runs. The transition velocities $\dot{\xi}(0)$ were sampled from a Gaussian distribution and according to Eq. (1.5) these velocities were used as weight factor in an average over all runs. In order to reduce the number of relaxation runs we here used the product of

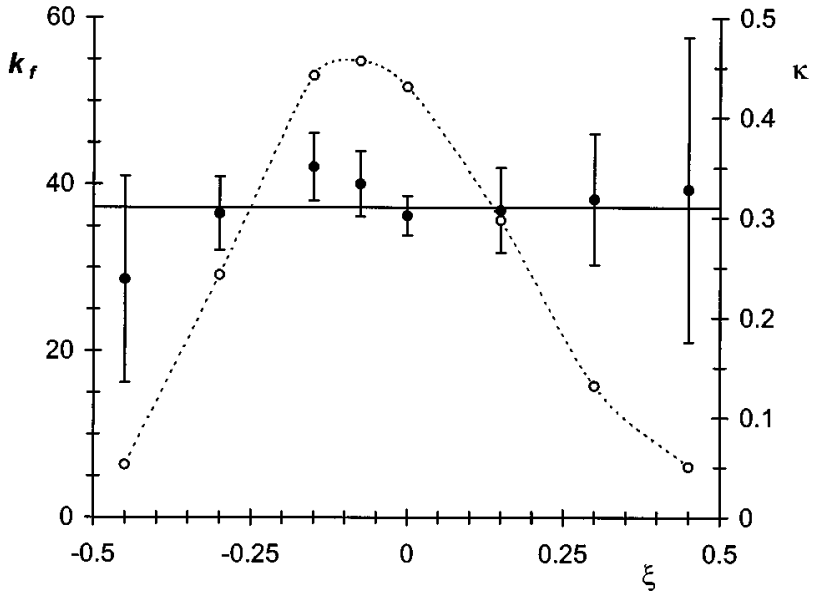

FIG. 5. Transmission function (open) and reactive flux rate (solid) as a function of the location of the transition state of a calix[4]arene in chloroform.

the Gaussian and the weight factor as the new probability distribution from which velocities were drawn. Between 500 and 1000 relaxations runs were found to suffice for an accurate calculation of the transmission coefficient. The effects of the transition state on the transmission function and the reactive flux rate are shown in Fig. 5. In the range of $\xi^{\neq}$ between +0.45 and -0.45 the transmission function is seen to vary by a factor of 10 . The reactive flux rate constant, on the other hand, is fairly constant.

Isomerization rates of calix[4]arenes with various sidegroups and in various solvents have been measured with ${ }^{1}$ H-NMR. ${ }^{21-24}$ With the calix[4]arene of this paper, however, comparison of the theoretical and experimental transition rates is complicated by the fact that the paco conformation is only very short-lived. Hence only the cone to inverted cone rate is experimentally accessible. This inversion results from a series of independent reactions with one phenyl ring flipping over in each step, ${ }^{18}$ as illustrated in Fig. 6. Because of symmetry, the flowchart can be reduced to

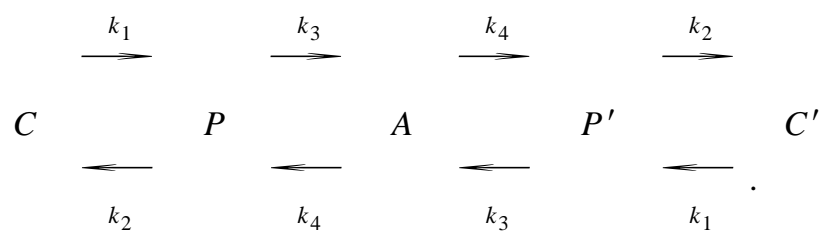

Here $C$ denotes the cone with all four phenyl rings pointing upwards, $P$ denotes the set of four conformations with three phenyl rings pointing upwards, $A$ denotes the set of six alternate conformations with two phenyl rings pointing upwards, and $C^{\prime}$ and $P^{\prime}$ are defined likewise as inverted paco and inverted cone. The five time constants of the relaxation processes of this system are obtained as the eigenvalues of the matrix of the transition probabilities. The relevant eigenvalue is 


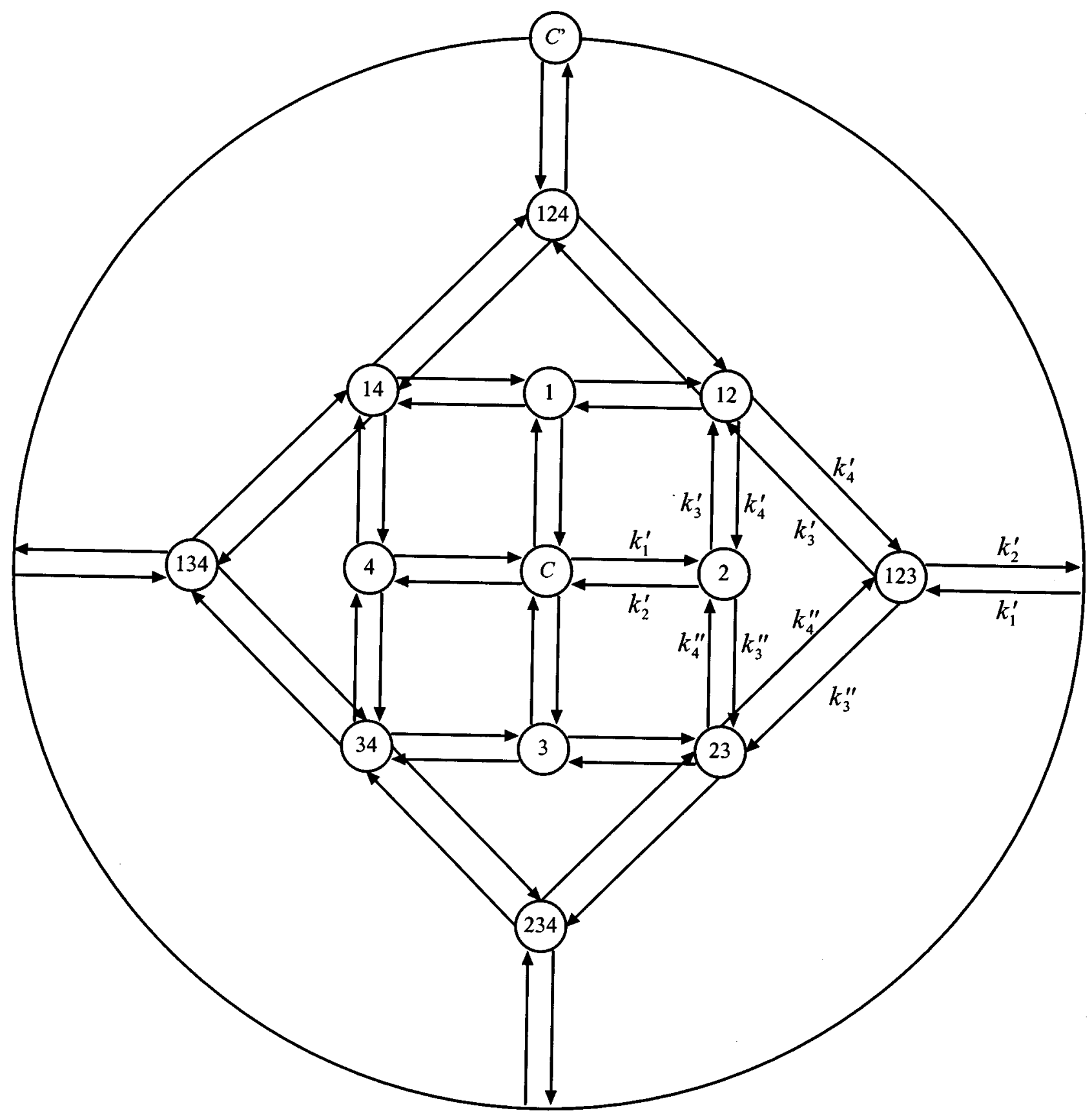

FIG. 6. Flowchart of the various stable conformations and the possible interconversions of a calix[4]arene. In the cone conformation (C) all phenyl rings are pointing upwards, in conformation " 1 " the ring numbered 1 is pointing downwards, etc. The entire outer circle corresponds to the inverted cone $\left(\mathrm{C}^{\prime}\right)$, in which all phenyls are pointing downward. The 1,3-alternate conformations are not shown.

$$
\begin{aligned}
k & =\frac{1}{2}\left[\left(k_{1}+k_{2}+k_{3}\right)-\sqrt{\left(k_{1}+k_{2}+k_{3}\right)^{2}-4 k_{1} k_{3}}\right] \\
& \approx k_{1} \frac{k_{3}}{k_{2}+k_{3}},
\end{aligned}
$$

where in the second line it is assumed that $k_{1}$ is much smaller than the other $k$ 's. The corresponding eigenvector describes the exchange of molecules between $C$ and $C^{\prime}$ with accompanying changes in $P$ smaller by a factor of $k_{1} /\left(k_{2}\right.$ $\left.+k_{3}\right)$ and no changes in A. In Fig. 6 the rates of going from one particular conformation (e.g., a paco with phenyl ring number 2 rotated) to another particular confirmation are marked by (double) primes. These primed rates can be calculated with the techniques described in this paper, while the unprimed rates are the experimentally accessible rates. ${ }^{24} \mathrm{We}$ now want to find a relation between $k$ and $k_{1}^{\prime}$.

There are four possible routes leading from the cone to the paco, so $k_{1}=4 k_{1}^{\prime}$. Rather than calculating $k_{2}$ and $k_{3}$ 
TABLE I. Computed and experimental reaction rates.

\begin{tabular}{lccr}
\hline \hline \multicolumn{1}{c}{ Method } & $k^{\mathrm{TST}} / \mathrm{s}^{-1}$ & $\kappa$ & $k^{\mathrm{RF} / \mathrm{s}^{-1}}$ \\
\hline Vacuum normal modes & 174 & & \\
Vacuum umbrella sampling, $t=0.6 \mathrm{ps}$ & 256 & 0.92 & 237 \\
Vacuum umbrella sampling, $t=2.0 \mathrm{ps}$ & 256 & 0.82 & 209 \\
Chloroform umbrella sampling & 84 & 0.43 & 36 \\
${ }^{1} \mathrm{H}-\mathrm{NMR}$ experiment & & & $7-63$ \\
\hline \hline
\end{tabular}

exactly, we will estimate their values from transition state theory based on normal-mode analysis. The rate of the paco to cone transition then is $k_{2}=k_{2}^{\prime}=1.1 \times 10^{8} \mathrm{~s}^{-1}, \Delta A^{\neq}=6.6$ $\mathrm{kcal} / \mathrm{mol}$. The rate of the paco to alternate transition equals the sum of the two different paco to 1,2-alternate transitions, $k_{3}=k_{3}^{\prime}+k_{3}^{\prime \prime}$, with $k_{3}^{\prime}=2.1 \times 10^{8} \mathrm{~s}^{-1}, \Delta A^{\neq}=6.2 \mathrm{kcal} / \mathrm{mol}$ and $k_{3}^{\prime \prime}=1.1 \times 10^{8} \mathrm{~s}^{-1}, \Delta A^{\neq}=6.6 \mathrm{kcal} / \mathrm{mol}$. With $\Delta A^{\neq}$ $=12.4 \mathrm{kcal} / \mathrm{mol}$, the paco to 1,3 -alternate transition is too slow to be of any influence. Substitution of these rates in the second line of Eq. (3.3) yields $k=2.99 k_{1}^{\prime}$, so as a rule of thumb the experimentally observed cone to inverted cone rate $k$ equals three times the cone to paco reaction rate $k_{1}^{\prime}$. Of course, this estimate should not be taken for granted, but it gives a reasonable approximation of the effect that is to be expected in exact calculations.

Gutsche and Bauer ${ }^{21}$ measured a $\Delta A^{\neq}$of $14.9 \mathrm{kcal} / \mathrm{mol}$, $k=189 \mathrm{~s}^{-1}$, at the coalescence temperature of $36^{\circ} \mathrm{C}$. Araki et $a .^{22}$ found a slightly higher coalescence temperature of $44{ }^{\circ} \mathrm{C}$, and a rate of $20 \mathrm{~s}^{-1}, \Delta A^{\neq}=15.7 \mathrm{kcal} / \mathrm{mol}$, at $25^{\circ} \mathrm{C}$. With the rule of thumb they yield a cone to paco rate $k_{1}^{\prime}$ of 63 and $7 \mathrm{~s}^{-1}$, respectively. These figures compare surprisingly well with the $36 \mathrm{~s}^{-1}$ calculated with the reactive flux method. The various rate constants are summarized in Table I.

\section{CONCLUSIONS}

The reaction coordinate based on the unstable normal mode at the saddle point of the potential energy surface is shown to be a very convenient reaction coordinate, both in the calculation of the probability distribution along the reaction coordinate and in the calculation of transmission coefficients. The free-energy function obtained by a normal-mode analysis as a function of $\xi$ proves to be an excellent first guess at the umbrella function. The reaction rates calculated with the reactive flux method for a calix[4]arene in vacuo and in chloroform are virtually independent of the chosen transition state. The isomerization rate of the solvated molecule is in good agreement with experimental data.

\section{APPENDIX: NORMAL-MODE ANALYSIS AND $Q(\xi)$}

In this Appendix we will calculate the partition function $Q\left(\xi^{*}\right)$, defined by Eq. (2.13), within the harmonic approximation. To this end we first locate the minimum energy configuration $\mathbf{R}^{*}$, as indicated in Sec. II B. We next perform a normal-mode analysis in the neighborhood of $\mathbf{R}^{*}$. We ap- proximate the hyperplane $\xi(\mathbf{X})=\xi^{*}$ by a tangent plane at $\mathbf{R}^{*}$. The unit vector normal to this hyperplane at $\mathbf{R}^{*}$ follows from the gradient of $\xi$, Eq. (2.7),

$$
\mathbf{N}=\frac{\mathbf{Q}^{r}-\sum_{k=1}^{3} d_{k}^{r} \mathbf{S}^{k}}{1+\sum_{k=1}^{3}\left(d_{k}^{r}\right)^{2}} \text {. }
$$

The tangent plane is spanned by a set of $3 N-1$ unit vectors perpendicular to $\mathbf{N}$,

$$
\mathbf{T}^{l}=\left\{\begin{array}{l}
\mathbf{E}^{k}, \quad k=1,2,3 \\
\mathbf{Q}^{j}, \quad j=1, \ldots, 3 N-6, \quad j \neq r, \\
\mathbf{S}^{k}+d_{k}^{r} \mathbf{Q}^{r}, \quad k=1,2,3
\end{array}\right.
$$

where the final three vectors still need to be orthonormalized. The eigenfrequencies of vibration, $\omega_{j}^{*}$, in the hyperplane

$$
\mathbf{X}=\mathbf{R}^{*}+\sum_{l=1}^{3 N-1} \tau_{l} \mathbf{T}^{l}
$$

are found by diagonalizing the $3 N-1$ dimensional Hessian at $\mathbf{R}^{*}$,

$$
H_{k l}^{*}=\frac{\partial^{2} \Phi}{\partial \tau_{k} \partial \tau_{l}} .
$$

Each $3 N-1$-dimensional eigenvector is next transformed into a $3 N$-dimensional atomic displacements vector by summing over the $\mathbf{T}^{l}$ using the components of the eigenvector as weight factors. The displacement vectors are again subdivided into three groups, the translational vectors $\mathbf{E}^{* l}$, identical to the $\mathbf{E} l$, and the rotational vectors $\mathbf{S}^{* k}$, of the form of Eq. (2.2) with $\mathbf{r}_{i}^{0}$ replaced by $\mathbf{r}_{i}^{*}$, all having eigenfrequency zero, and the vibrational vectors $\mathbf{Q}^{* j}$ all having positive eigenfrequencies. Analogously to Eq. (2.6), we now express any configuration as

$$
\mathbf{X}=\mathbf{A}^{*} \mathbf{R}+\sum_{l=1}^{3} \gamma_{l}^{*} \mathbf{E}^{* l}, \quad \mathbf{R}=\mathbf{R}^{*}+\sum_{j=1}^{3 N-7} \alpha_{j}^{*} \mathbf{Q}^{* j}+\beta^{*} \mathbf{N},
$$

where the rotation matrix $\mathbf{A}^{*}$ is determined by

$$
0=\left(\mathbf{X}-\mathbf{A}^{*} \mathbf{R}^{*}\right) \cdot \mathbf{A}^{*} \mathbf{S}^{* k}, \quad k \in\{1,2,3\} .
$$

It is obvious that $\xi(\mathbf{X})$ (with $\xi$ still defined with respect to $\mathbf{R}^{0}$ ) is a function of the $\alpha_{j}^{*}$ and $\beta^{*}$ only, and does not depend on $\mathbf{A}^{*}$. Because of the construction of the $\mathbf{Q}^{* j}$, we have

$$
\xi(\mathbf{X})=\xi^{*}+\beta^{*}\left|\nabla_{\mathbf{R}} \xi\right|,
$$

up to first order in $\alpha_{j}^{*}$ and $\beta^{*}$, where it was used that $\mathbf{N}$ is a unit vector parallel to $\nabla_{\mathbf{x}} \xi$.

The integrals of the partition function in Eq. (2.13) are solved analogously to those in Eq. (2.8). The sole exception is the integral over $\beta^{*}$ which, because of the delta function, yields not a frequency factor but $\left|\nabla_{\mathbf{R} *} \xi\right|^{-1}$, with

$$
\left|\nabla_{\mathbf{R} *} \xi\right|=\left[\sum_{i=1}^{N}\left(\frac{\partial \xi}{\sqrt{m_{i}} \partial \mathbf{x}_{i}}\right)^{2}\right]^{1 / 2}=\left[1+\sum_{k=1}^{3}\left(d_{k}^{r}\right)^{2}\right]^{1 / 2} .
$$

We then arrive at 


$$
\begin{aligned}
Q\left(\xi^{*}\right)= & \left(\frac{2 \pi M k T}{h^{2}}\right)^{3 / 2} V \cdot \frac{\pi^{1 / 2}}{\sigma}\left(\frac{8 \pi^{2} k T}{h^{2}}\right)^{3 / 2}\left(I_{A}^{*} I_{B}^{*} I_{C}^{*}\right)^{1 / 2} \\
& \times \prod_{i=1}^{3 N-7} \frac{k T}{\hbar \omega_{i}^{*}} \cdot \omega_{e} e^{-E^{*} / k T} \cdot\left|\nabla_{\mathbf{R}^{*}} \xi\right|^{-1} \\
& \cdot(2 \pi k T)^{1 / 2} h^{-1} .
\end{aligned}
$$

We end this appendix with the derivation of an alternative expression for the above integral. Rather than introducing a new set of coordinates based on $\mathbf{R}^{*}$, we keep on using the coordinates defined with respect to $\mathbf{R}^{0}$. The delta function in the partition function is then trivially dealt with. The Jacobi matrix of the transformation from mass-weighted coordinates to generalized coordinates is again given by Eq. (2.11). However, for small vibrations around $\mathbf{R}^{*}$ the approximation of $\mathbf{R}$ by $\mathbf{R}^{*}$ leads to three vectors $\epsilon^{k} \mathbf{R}^{*}$ that are no longer orthogonal to the $\mathbf{Q}^{j}$. Expressing these vectors in the $\mathbf{R}^{0}$-based vectors yields

$$
\begin{aligned}
& \mathbf{J}=\mathbf{A}\left(\begin{array}{lll}
\epsilon^{k} \mathbf{R}^{0} & \mathbf{Q}^{j} & \mathbf{A}^{-1} \mathbf{E}^{l}
\end{array}\right)\left(\begin{array}{ccc}
\left(\mathbf{I}^{0}\right)^{-1} \sigma^{*} & \mathbf{0} & \mathbf{0} \\
\rho^{*} & \mathbf{1} & \mathbf{0} \\
\mathbf{0} & \mathbf{0} & \mathbf{1}
\end{array}\right) \\
& \times\left(\begin{array}{ccc}
\mathbf{c} & \mathbf{0} & \mathbf{0} \\
\mathbf{0} & \mathbf{1} & \mathbf{0} \\
\mathbf{0} & \mathbf{0} & \mathbf{1}
\end{array}\right), \\
& |\mathbf{J}|=\left|\sigma^{*}\right|\left(I_{A}^{0} I_{B}^{0} I_{C}^{0}\right)^{-1 / 2} \sin \psi_{2},
\end{aligned}
$$

where

$$
\sigma_{l k}^{*}=\left(\epsilon^{l} \mathbf{R}^{*}\right) \cdot\left(\epsilon^{k} \mathbf{R}^{0}\right), \quad \rho_{l j}^{*}=\left(\epsilon^{l} \mathbf{R}^{*}\right) \cdot \mathbf{Q}^{j},
$$

and the $\left(\mathbf{I}^{0}\right)^{-1}$ arises because the $\epsilon^{k} \mathbf{R}^{0}$ are not orthogonal. Performing the integration over the generalized coordinates and multiplying with the integral over the momenta gives

$$
\begin{aligned}
Q\left(\xi^{*}\right)= & \left(\frac{2 \pi M k T}{h^{2}}\right)^{3 / 2} V \cdot \frac{\pi^{1 / 2}}{\sigma}\left(\frac{8 \pi^{2} k T}{h^{2}}\right)^{3 / 2}\left(I_{A}^{0} I_{B}^{0} I_{C}^{0}\right)^{-1 / 2} \\
& \times \prod_{i=1}^{3 N-7} \frac{k T}{\hbar \omega_{i}^{* *}} \cdot \omega_{e} e^{-E^{* / k T}} \cdot\left|\sigma^{*}\right|(2 \pi k T)^{1 / 2} h^{-1} .
\end{aligned}
$$

The required eigenfrequencies are found as the square roots of the eigenvalues of the $3 N-7$-dimensional Hessian at $\mathbf{R}^{*}$,

$$
H_{j k}^{* *}=\frac{\partial^{2} \Phi}{\partial \alpha_{j} \partial \alpha_{k}}, \quad j, k \neq r,
$$

and differ from the eigenfrequencies used in Eq. (2.15).

${ }^{1}$ H. Eyring, J. Chem. Phys. 3, 107 (1935).

${ }^{2}$ P. Hänggi, P. Talkner, and M. Borkovec, Rev. Mod. Phys. 62, 251 (1990).

${ }^{3}$ T. Yamamoto, J. Chem. Phys. 33, 281 (1960).

${ }^{4}$ D. Chandler, J. Chem. Phys. 68, 2959 (1978).

${ }^{5}$ W. K. den Otter and W. J. Briels, J. Chem. Phys. 106, 5494 (1997).

${ }^{6}$ W. H. Miller, Acc. Chem. Res. 9, 306 (1976).

${ }^{7}$ D. A. McQuarrie, Statistical Mechanics (Harper and Row, New York, 1976).

${ }^{8}$ H. Goldstein, Classical Mechanics (Addison-Wesley, Reading, MA, 1980).

${ }^{9}$ J. Ryckaert, G. Ciccotti, and H. J. C. Berendsen, J. Comput. Phys. 23, 327 (1977).

${ }^{10}$ D. L. Beveridge and F. M. DiCapua, Annu. Rev. Biophys. Biophys. Chem. 18, 431 (1989).

${ }^{11}$ W. F. van Gunsteren, T. C. Beutler, F. Fraternali, P. M. King, A. E. Mark, and P. E. Smith in Computer Simulations of Biomolecular Systems: Theoretical and Practical Applications, edited by W. F. van Gunsteren, P. K. Weiner, and A. J. Wilkinson (ESCOM, Leiden, The Netherlands, 1993), p. 315.

${ }^{12}$ T. P. Straatsma, Rev. Comp. Chem. 9, 81 (1996).

${ }^{13}$ J.-M. Depaepe, J.-P. Ryckaert, E. Paci, and G. Ciccotti, Mol. Phys. 79, 515 (1993).

${ }^{14}$ R. W. W. Hooft, B. P. van Eijck, and J. Kroon, J. Chem. Phys. 97, 6690 (1992).

${ }^{15}$ S. Kumar, D. Bouzida, R. H. Swendsen, P. A. Kollman, and J. M. Rosenberg, J. Comp. Chem. 13, 1011 (1992).

${ }^{16}$ C. D. Gutsche, J. S. Rogers, D. Stewart, and K.-A. See, Pure \& Appl. Chem. 62, 485 (1990).

${ }^{17}$ H. J. C. Berendsen and W. F. van Gunsteren, GROMOS Reference Manual (University of Groningen Press, Groningen, The Netherlands, 1987).

${ }^{18}$ S. Fischer, P. D. J. Grootenhuis, L. C. Groenen, W. P. van Hoorn, F. C. J. M. van Veggel, D. N. Reinhoudt, and M. Karplus, J. Am. Chem. Soc. 117, 1611 (1995)

${ }^{19}$ S. Fischer and M. Karplus, Chem. Phys. Lett. 194, 252 (1992).

${ }^{20}$ S. Glasstone, K. J. Laidler, and H. Eyring, The Theory of Rate Processes (McGraw-Hill, New York, 1941).

${ }^{21}$ C. D. Gutsche and L. J. Bauer, J. Am. Chem. Soc. 107, 6052 (1985).

${ }^{22}$ K. Araki, S. Shinkai, and T. Matsuda, Chem. Lett. 1989, 581 (1989).

${ }^{23}$ W. P. van Hoorn, W. J. Briels, J. P. M. van Duynhoven, F. C. J. M. van Veggel, and D. N. Reinhoudt, J. Am. Chem. Soc. (submitted).

${ }^{24}$ J. Blixt and C. Detellier, J. Am. Chem. Soc. 116, 11957 (1994). 